\title{
Synthetic pyrethroid insecticides: a dermatological evaluation
}

\author{
S A FLANNIGAN,' S B TUCKER,' M M KEY,' C E ROSS, ${ }^{2}$ E J FAIRCHILD II,' \\ B A GRIMES,' AND R B HARRIST'
}

From the University of Texas ${ }^{1}$ Health Science at Houston, Schools of Medicine and Public Health, Houston, Texas, and Corporate Medical Department, ${ }^{2}$ Shell Oil Company, Houston, Texas, USA

ABSTRACT Synthetic pyrethroids are lipophilic insecticides whose biological activity seems to be directly related to their chemical structure. In this investigation differences in cutaneous sensation were detected by human participants between synthetic pyrethroids with a cyano group in the (S)-configuration of the 3-phenoxybenzyl alcohol of their molecular structure (fenvalerate) and those that do not (permethrin). A strong relation was noted between insecticidal potency and degree of induced cutaneous sensation for the alpha-cyano and non-cyano pyrethroids, with a prominent difference between the two. No sensation was observed by any of the same participants on topical exposure to the inert ingredients of these agents. A linear correlation between concentration and degree of induced dysaesthesia was observed for both pyrethroids. Regressing the cutaneous sensation on the common logarithm of concentration resulted in a regression equation of $\mathrm{Y}=84 \cdot 0+31 \cdot 0 \mathrm{X}_{1}$ for fenvalerate and $\mathrm{Y}=27 \cdot 5+15 \cdot 8 \mathrm{X}_{1}$ for permethrin. A highly efficacious therapeutic agent for pyrethroid exposure was noted to be dl-alpha tocopherol acetate. An impressive degree of inhibition of paraesthesia resulted from the topical application of vitamin $\mathrm{E}$ acetate, with a therapeutic index of almost $100 \%$.

Synthetic pyrethroid insecticides have evolved through a classic sequence of events: activity was first noticed in a natural extract from the flowers of Chrysanthemum cinerariaefolium; reactive agents were isolated and identified; and finally, increasingly active analogues were synthesised. Few insecticides are as biologically active or possess such a great potential for structural variation with total retention, or even possibly enhancement, of potency as the synthetic pyrethroids. They incorporate a high insecticidal activity (knockdown) for a wide variety of insects with a relatively low toxicity for mammals. Numerous derivatives of the original pyrethroids are now among the most potent pesticides known to man and are being evaluated for many different applications, as well as for possible replacements of the organophosphate, carbamate, and organochlorine insecticides.' Well over 1000 pyrethroids have been synthesised to date.

The natural pyrethrins have long been used against a wide variety of garden and horticultural pests, but their poor residual capability, combined

Received 21 May 1984

Accepted 2 July 1984 with a high cost, restricted their agricultural application. ${ }^{2}$ The more recently synthesised pyrethroids, however, combine this high insecticidal activity with a persistence necessary to control a broad range of pests. A better knowledge of the field behaviour of the synthetic pyrethroids in relation to the pest/ predator complex of each agricultural region has shown how judicious applications can actually minimise harm to beneficial insects. Physical properties, such as involatility, lipophilicity, and susceptibility to degradation by micro-organisms, have severely restricted the efficaciousness of these agents against various soil insects.

The biological activity of the synthetic pyrethroid insecticides seems to be directly related to their chemical structure.$^{34}$ Almost all active pyrethroids are esters of cyclopropane carboxylic acids with alkenylmethyl cyclopentenolone alcohols. Their constituent acids and alcohols, and simple derivatives, are practically inactive. Properties such as electron density and polarisability are of little importance. The alpha-cyano derivatives, with a cyano group in the S-configuration of the 3-phenoxybenzyl alcohol of the molecular structure, have an increased potency of approximately four- 
fold with insects. ${ }^{3} \mathrm{~A}$ higher toxicity for cis-isomers, as compared with their corresponding trans-isomers, has also been observed for numerous pyrethroids in the rat. The highly lipophilic nature of the synthetic pyrethroids has made them practically insoluble in water, a property that promotes rapid penetration through an insect's cuticle.

Synthetic pyrethroids are considered broad range insecticides that are nerve toxins. They act directly on the axon through interference with the sodium channel gating mechanism that underlies the generation and conduction of each nerve impulse. ${ }^{56}$ The pyrethroids induce the sodium channels to close slower than normal, resulting in a gradually decaying inward sodium current after termination of membrane depolarisation. This slow influx of sodium ions on termination of depolarisation is referred to as a sodium tail current. Non-cyano pyrethroids induce tail currents with time constants of decay in the order of milliseconds, whereas alpha-cyano pyrethroids result in time constants of decay in the hundreds of milliseconds to more than one second.

Recent clinical studies with isolated preparations of the peripheral nervous system of the clawed frog, Xenopus laevis, have augmented the distinction between two main classes of these synthetic insecticides:

(1) Pyrethroids which do not have an alpha-cyano group attached to their molecular structure (allethrin, permethrin, cismethrin, and bioresmethrin). These particular insecticides result in relatively short trains of nerve impulses in the lateral line sense organ. In the peripheral nerves they induce a depolarising after potential with repetitive firing.

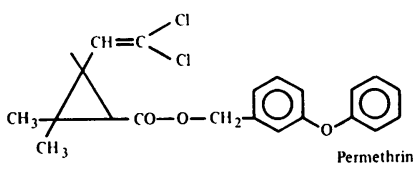

(2) Pyrethroids with an alpha-cyano group attached to the 3-phenoxybenzyl alcohol of the molecular structure (deltamethrin, cypermethrin, fenvalerate, and fenpropathrin). These pyrethroids induce long trains of nerve impulses in the lateral line sense organ and do not invoke repetitive activity in the peripheral nerves. Instead, they cause a quickly reversible, stimulus frequency dependent suppression of the nerve action potential, resulting in a long lasting depolarising after potential. ${ }^{78}$

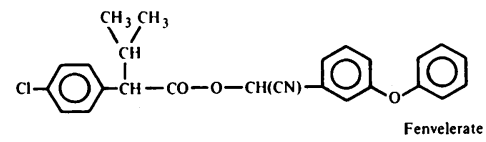

People are capable of tolerating greater exposures to synthetic pyrethroids than other insecticides, making them a welcome option. Toxicity from inhalation or oral or dermal contact with these agents are all minimal. They are not teratogenic in rats, mice, or rabbits nor mutagenic with select bacterial strains. Subacute and chronic feeding of greater quantities of these agents to rats has invariably caused some histopathological changes in the liver; however, these studies are neither indicative nor suggestive of tumorigenicity. ${ }^{9}$ The relatively low inherent toxicity witnessed in mammals may be attributed to various factors preventing direct interaction with the nervous system. Unlike other insecticides, they have numerous sites for metabolic degradation. ${ }^{10}$ One route involves ester cleavage, often controlled by the degree of stearic hindrance about the linkage. Numerous sites on the molecules are also susceptible to attack by mixed function oxidase systems. Therefore, their lipophilic characteristic enhances rapid penetration to the arthropod's nervous system, whereas in mammals they are biodegraded both oxidatively and hydrolytically, depending on the chemical composition of the pyrethroid. Rapid metabolic degradation, together with incomplete absorption from the gastrointestinal tract, accounts for the low acute toxicity of these insecticides. This has been substantiated by their increased toxic nature when administered to mammals intravenously rather than orally." ${ }^{11}$ Even myelinated nerves of vertebrates are thought to sequester the pyrethroid molecules, known to be lipid soluble in the myelin sheath, thereby preventing a portion of their chemical effect on the nerve axon. ${ }^{12}$ The accumulation of the pyrethroids, however, and their degradation products in various tissues over time has not been fully evaluated. Consequently, further chronic toxicity studies are warranted to ascertain the final destination of these molecules after exposure.

At present, there is little information in the occupational medical journals concerning the biological ramifications of human cutaneous exposure to the synthetic pyrethroids. Kolmodin-Hedman et al noted that forestry workers displayed symptoms that were mainly irritative, such as itching and burning of the skin. ${ }^{13}$ Some individuals reported cough and dyspnoea when using fenvalerate and permethrin as a wettable powder. The authors indicated that whether the burning and tingling skin sensation reported by some of the subjects was an irritative one, or a sign of peripheral sensory nerve involvement, could not be discerned. LeQuesne et al found that among 23 research workers exposed to synthetic pyrethroids at the Shell Research Laboratories at Sittingbourne, 19 experienced one or more episodes 
of abnormal facial sensation, developing with a latent period of 30 minutes to three hours and persisting for about 30 minutes to eight hours. ${ }^{14}$ They noted that the delay in the onset of symptoms after exposure was unlike that after cutaneous contact with any other irritant solution. There were no abnormal neurological signs, and electrophysiological studies were normal in the arms and legs of each worker. Tucker and Flannigan noted a difference in the degree of paraesthesia among agricultural applicators of fenvalerate. ${ }^{15}$ The susceptibility of the workers did not appear to be based on race, personal history of atopy, or the number of exposures. Virtually all exposed individuals were affected. The cutaneous sensation described was a stinging or burning that progressed to numbness in about one third of the exposed workers. The sensation typically began several hours after contact, peaked in the evening, and was rarely present the next morning. The intensity of the sensation varied according to the type and extent of exposure. Clinical signs of inflammation, such as oedema or vesiculation, were not apparent. The response did not increase with continued exposure in a given season or even over several seasons, and did not appear to decrease in sensitivity, as one might expect in a tachyphylactic response when using a compound on a daily basis.

As early as 1941, workers with the plant extract, pyrethrum, noted a paraesthesia similar to, but less intense than, that described by present day workers with the more potent synthetic agents. ${ }^{16}$ The universal aspect of this cutaneous reaction is emphasised by reports from diverse parts of the world, as well as from varying occupational settings, indicating that paraesthesia from the pyrethroids is a widespread problem. ${ }^{13-16}$ The sensation can be quite uncomfortable, and may result in the substitution of more toxic insecticides by sensitive workers in order to avoid the annoying paraesthesia. Even mild sensations may lead to pronounced anxiety. To summarise, the occurrence of paraesthesia is widespread and may result in discomfort and anxiety leading to a decrease in the usefulness of this class of insecticide, despite several advantages over other insecticides which cause no apparent cutaneous sensation.

\section{Materials and methods}

The following studies were conducted to test the hypothesis that possible differences between two widely used synthetic pyrethroids exist in producing cutaneous sensation or irritation or both. One of the two agents evaluated was fenvalerate, an alphacyano pyrethroid, the other being permethrin, a non-cyano pyrethroid. The chemical name for fenvalerate is cyano (3-phenoxyphenyl) methyl 4-chloro-alpha-(1-methylethyl) benzeneacetate, with an empirical formula of $\mathrm{C}_{25} \mathrm{H}_{22} \mathrm{ClNO}_{3}$. Trade names for fenvalerate are Sumicidin, Belmark, and Pydrin. Permethrin's chemical name is (3phenoxyphenyl) methyl ( \pm ) cis-trans 3(2-2-dichloroethenyl)-2-, 2-dimethyl-cyclopropanecarboxylate and has an empirical formula of $\mathrm{C}_{21} \mathrm{H}_{20} \mathrm{Cl}_{2} \mathrm{O}_{3}$. Trade names for permethrin are Adion, Ambush, Ectiban, Kafil, Matadan, Perthrine, Pounce, and Talcord. Two chemical grades of both insecticides were evaluated in this investigation. The technical grade of either pyrethroid was composed of approximately $94-96 \%$ active ingredient, whereas the formulated grade consisted of $32-36 \%$ active ingredient. The remainder of either grade, of both pyrethroids, entailed similar inert ingredients (organic solvents and surfactants).

All portions of this investigation have been reviewed and approved by the University of Texas at Houston Committee for the Protection of Human Subjects. The animal study was also reviewed and deemed acceptable by the Health Science Center Animal Shelter Committee. Therefore, based on numerous earlier investigations and the high dermal $\mathrm{LD}_{\mathbf{s o}}$, the following studies were conducted.

STUDY 1: COMPARISON OF INDUCED PARAESTHESIA FROM FENVALERATE, PERMETHRIN, OR THE INERT INGREDIENTS

Clinically, a comparison was made of the capability of human participants to discriminate between the dysaesthesia induced by a technical or formulated alpha-cyano pyrethroid (Fenvalerate), a technical or formulated non-cyano pyrethroid (permethrin), and the inert ingredients that comprise a major portion of both formulated compounds. Both technical pyrethroids were diluted with absolute ethanol whereas the formulated compounds were diluted in distilled water. The evaluation used a previously developed assay..$^{17} 18$ The participants were exposed to the cutaneous sensation before the onset of the study. Preparation consisted of two separate topical applications, one with a field strength alpha-cyano pyrethroid $\left(0.13 \mathrm{mg} / \mathrm{cm}^{2}\right)$ and the other with a field strength non-cyano pyrethroid $\left(0.13 \mathrm{mg} / \mathrm{cm}^{2}\right)$, with a review and discussion of the paraesthesia felt by each subject. The same technique was used for all participants in each portion of this research. Concentrations were prepared and recorded throughout the investigation in $\mathrm{mg} / \mathrm{cm}^{2}$ which pertains to the number of milligrams of synthetic pyrethroid topically applied per unit area of skin.

Application of $0.05 \mathrm{cc}$ of field strength formulated or technical fenvalerate $\left(0.13 \mathrm{mg} / \mathrm{cm}^{2}\right)$, field strength formulated or technical permethrin 
$\left(0.13 \mathrm{mg} / \mathrm{cm}^{2}\right)$, or the inert ingredients were delivered to a $4 \mathrm{~cm}^{2}$ area on the lower margin of one earlobe of each participant via a tuberculin syringe (accuracy $\pm 0.002 \mathrm{cc}$ ) on five separate occasions. The opposite earlobe received $0.05 \mathrm{cc}$ of distilled water when opposing a formulated pyrethroid and absolute ethanol as the control for a technical pyrethroid. Participant evaluation after each application continued for 48 hours and involved grading and describing any unusual cutaneous sensations. The grading scale was on a basis of 0 to 4 , with $0=$ no sensation; $1=$ mildly felt sensation; 2 = definite sensation, no discomfort; $3=$ moderate sensation, little or no discomfort; and $4=$ intense sensation with discomfort. All applications were made in a double blind fashion from numbered vials. Each participant was therefore treated with only one of the five possible agents on each test date, with the vehicle always applied to the opposite ear. About 48 hours after each application, the same participant was treated with one of the remaining compounds on the opposite earlobe from the preceding application. Earlier investigations showed that there were no significant carry over effects- that is, prior applications of a pyrethroid to an earlobe did not influence later applications to the same earlobe. ${ }^{18}$ This procedure was followed until each participant had evaluated both formulated and technical pyrethroids, and the inert ingredients. All treatments and sites were randomly assigned before application.

When comparing the overall cutaneous sensation from the various compounds, the aggregate of the reaction scores contained the same number of subjects, applications, and days applied. Since the unique characteristics of each individual remained constant under the different treatments, observations by each person tended to be correlated. The data over 48 hours for each participant were summarised, averaged, and tabulated. The results were analysed using an analysis of variance (ANOVA) with a repeated measure design, incorporating three cells (treatment levels) with six observations (subjects) per cell. ${ }^{19} 20$

To rank the three treatments, composed of either the inert ingredients, formulated fenvalerate and formulated permethrin, or the inert ingredients, technical fenvalerate and technical permethrin, a least significant difference (LSD) multiple comparison test was used if the overall $F$ test of the treatment differences was significant. The LSD test was chosen since it has good control of type I errors (rejecting the null hypotheses when the null hypotheses is correct) and more power than the methods of Newman-Keuls, Tukey, and Scheffe. ${ }^{21}$

Variations within each pyrethroid classification were evaluated by using an ANOVA with a repeated measure design, incorporating two cells with six observations per cell. Comparisons were made between either formulated and technical fenvalerate or formulated and technical permethrin.

STUDY 2: DIFFERENCES IN THE DOSE-RESPONSE CURVES FOR FENVALERATE AND PERMETHRIN

The dose-response curves for a formulated alphacyano pyrethroid (fenvalerate) and for a formulated non-cyano pyrethroid (permethrin), using the above assay on people, was assessed. For each participant, $0.05 \mathrm{cc}$ of a given dilution of a synthetic pyrethroid was applied to a $4 \mathrm{~cm}^{2}$ area on the lower margin of an earlobe through a tuberculin syringe (accuracy \pm $0.002 \mathrm{cc}$ ). The opposite earlobe was treated with distilled water (vehicle) and acted as the control. Evaluation by any participant continued for 48 hours and included grading and describing any unusual cutaneous sensation. All treatments were made in a double blind fashion from numbered vials that were randomly assigned before application. Dilutions of each formulated pyrethroid consisted of $2 \cdot 69,1 \cdot 00,0.36,0 \cdot 13$, and $0.01 \mathrm{mg} / \mathrm{cm}^{2}$. Each successive application was applied about 48 hours after the prior treatment on the opposite earlobe from the preceding application. Each participant's data for a given concentration of a pyrethroid were summed and tabulated. Columns were constructed proceeding from the lowest to the highest concentration of each pyrethroid for each participant. Columns of corresponding concentrations for each pyrethroid per participant were joined and normalised. The columns were then divided into separate pyrethroids, averaged over all participants and graphed on linear, semilog, and log paper to find the most appropriate linear fit for cutaneous sensation $v$ concentration. A regression analysis was calculated for both formulated fenvalerate and formulated permethrin by regressing the cutaneous sensation on the concentration of pyrethroid. A final plot consisted of both regression equations and their corresponding $95 \%$ confidence bands as calculated using the predicted mean values for either synthetic pyrethroid.

STUDY 3: THERAPEUTIC BENEFIT OF VITAMIN E ACETATE FOR SYNTHETIC PYRETHROID EXPOSURE

The therapeutic capability of topical vitamin $E$ acetate (dl-alpha tocopheryl acetate) to ameliorate the paraesthesia that accompanies cutaneous exposure to a technical or formulated alpha-cyano pyrethroid (fenvalerate) and to a technical or formulated noncyano pyrethroid (permethrin) was evaluated. The investigation used the aforementioned ear assay, with the same grading scale of 0 to 4 . 
Six volunteers were randomly treated with $0.05 \mathrm{cc}$ of either field strength technical or formulated fenvalerate $\left(0.13 \mathrm{mg} / \mathrm{cm}^{2}\right)$ or with technical or formulated permethrin $\left(0.13 \mathrm{mg} / \mathrm{cm}^{2}\right)$, applying each on a separate occasion to both the right and left earlobes. Approximately $0.05 \mathrm{cc}$ of vitamin $\mathrm{E}$ acetate $(1 \mathrm{mg}=$ 1 IU) was then randomly applied to either the right or left earlobe of each participant, allowing each subject to act as his own control. Evaluation continued for 48 hours and consisted of grading and describing any unusual cutaneous sensations. One of the remaining synthetic pyrethroids was then evaluated through the same format outlined above about 48 hours later. This procedure was followed until all participants had evaluated both formulated and technical fenvalerate, as well as formulated and technical permethrin. All applications were in a double blind fashion from numbered vials whereas treatments and sites were randomly assigned to participants before application.

The data acquired during the four evaluation periods, for each participant, were then summed, averaged, and tabulated. A therapeutic index was calculated for each synthetic pyrethroid using the equation:

Therapeutic index $\left.=100-\left[\begin{array}{l}\text { average } \\ \text { score of therapeutic } \\ \text { pyrethroid }+ \text { agent } \\ \begin{array}{l}\text { average score of } \\ \text { pyrethroid alone }\end{array}\end{array}\right] 100\right]$ An ANOVA with a repeated measure design, incorporating two cells with six observations per cell, was calculated on the averaged data to determine if a significant difference $(p \leqslant 0.05)$ actually existed between sites topically treated with or without dl-alpha tocopheryl acetate to ameliorate the cutaneous sensation of a synthetic pyrethroid.

\section{Results}

Figures 1 and 2 give the results of study 1 comparing the induced paraesthesia occurring from the topical application of fenvalerate, permethrin, or the inert ingredients. Figure 1 displays a prominent increase in paraesthesia between formulated fenvalerate $\left(0.13 \mathrm{mg} / \mathrm{cm}^{2}\right)$ and formulated permethrin $\left(0.13 \mathrm{mg} / \mathrm{cm}^{2}\right)$. No cutaneous sensation was noted by any of the participants to the inert ingredients (organic solvents and surfactants) when applied separately. The sensation developed with a latent period of about 30 minutes, peaked by eight hours and deteriorated as early as 24 hours. Comparison of the area under each curve shows an approximate fourfold difference in paraesthesia between the two synthetic pyrethroids. There was no statistically significant difference $(p \geqslant 0.05)$ in sensation be- tween the inert ingredients and formulated permethrin (table 1). Nevertheless, there was a significant difference between formulated fenvalerate and formulated permethrin, and between formulated fenvalerate and the inert ingredients.

The degree of induced paraesthesia for technical fenvalerate $\left(0.13 \mathrm{mg} / \mathrm{cm}^{2}\right)$, technical permethrin $\left(0.13 \mathrm{mg} / \mathrm{cm}^{2}\right)$, and the inert ingredients is displayed in fig 2. Again, an approximate fourfold increase can be noted between technical permethrin and technical fenvalerate, with the inert ingredients not being detected. The time course for cutaneous sensation

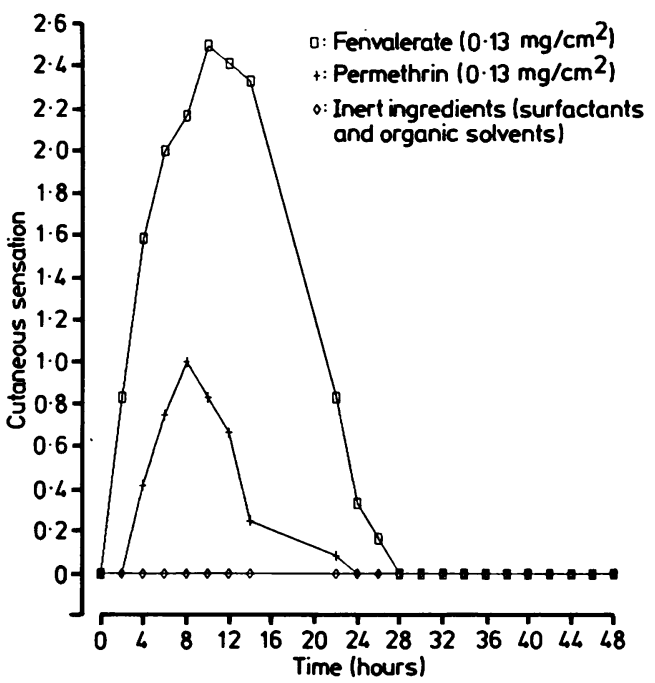

Fig 1 Paraesthesia resulting from formulated fenvalerate, formulated permethrin, and the inert ingredients on people.

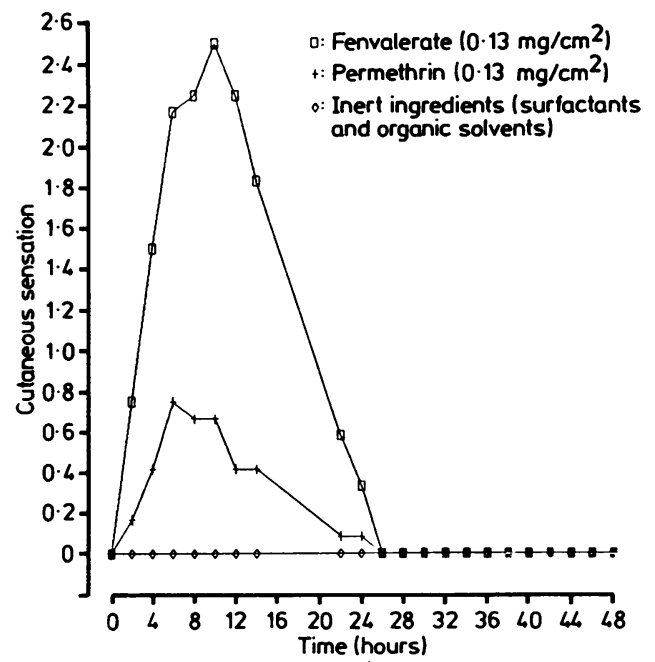

Fig 2 Paraesthesia resulting from technical fenvalerate, technical permethrin, and the inert ingredients on people. 
was similar to that for the formulated synthetic pyrethroids. There was a statistically significant difference between technical fenvalerate and technical permethrin, and between technical fenvalerate and the inert ingredients (table 1). As noted with the formulated compounds, there was little difference between technical permethrin and the inert ingredients. Interestingly, a significant difference did not exist between formulated and technical fenvalerate, nor between formulated and technical permethrin, when evaluated at the same concentration of $0 \cdot 13 \mathrm{mg} / \mathrm{cm}^{2}$.

Study 2 compared differences in the doseresponse curves for fenvalerate and permethrin using formulated fenvalerate and formulated permethrin at successive concentrations of $2 \cdot 69,1 \cdot 00$, $0.36,0.13$, and $0.01 \mathrm{mg} / \mathrm{cm}^{2}$. The most appropriate linear fit for both pyrethroid insecticides was on semilog paper with cutaneous sensation on the ordinate axis $(Y)$ and the common logarithm of concentration on the abscissa (X).

The correlation coefficient $(R)$ of the average cutaneous sensation noted by all participants for formulated fenvalerate and the common logarithm of concentration was $99.0 \%$. Regressing cutaneous sensation on the common logarithm of concentration resulted in a regression equation of $Y=84.0+$ $31 \cdot 0 \mathrm{X}_{1}$, with a square of the correlation coefficient $\left(R^{2}\right)$ of $84.9 \%$. The $R$ value for the average cutaneous sensation felt by all participants for formulated permethrin and the common logarithm of concentration was $94.0 \%$. Regressing the cutaneous sensation on the common logarithm of concentration resulted in a regression equation of $\mathrm{Y}=27.5+$ $15 \cdot 8 X_{1}$, with an $R^{2}$ value of $75 \cdot 6 \%$. Figure 3 presents the regression equations for formulated fenvalerate and formulated permethrin with $95 \%$ confidence bands appended to both. Comparing the equations, the $y$-intercept for fenvalerate is approximately threefold greater than for permethrin, whereas the slope $(\mathrm{m})$ is roughly twice as large.

Study 3 entailed the evaluation of the therapeutic benefit of dl-alpha tocopheryl acetate $(1 \mathrm{mg}=1 \mathrm{IU})$ for the dysaesthesia that results from topical expos-

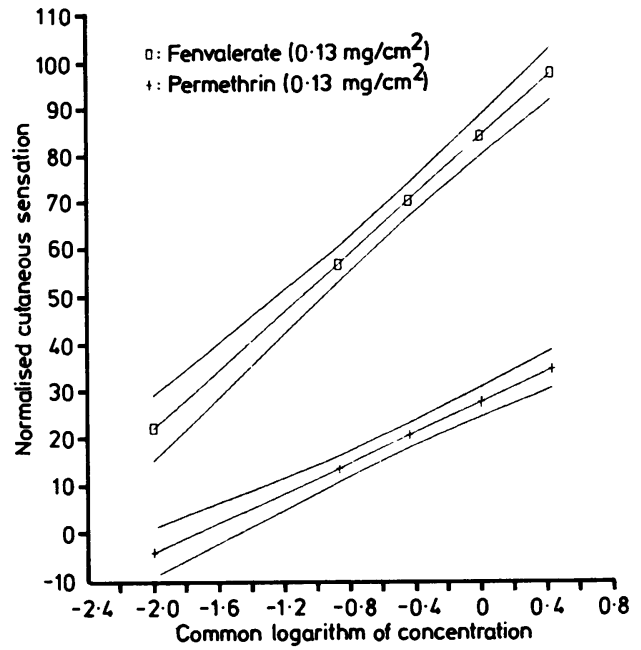

Fig 3 Regression equations for formulated fenvalerate and formulated permethrin with $95 \%$ confidence bands.

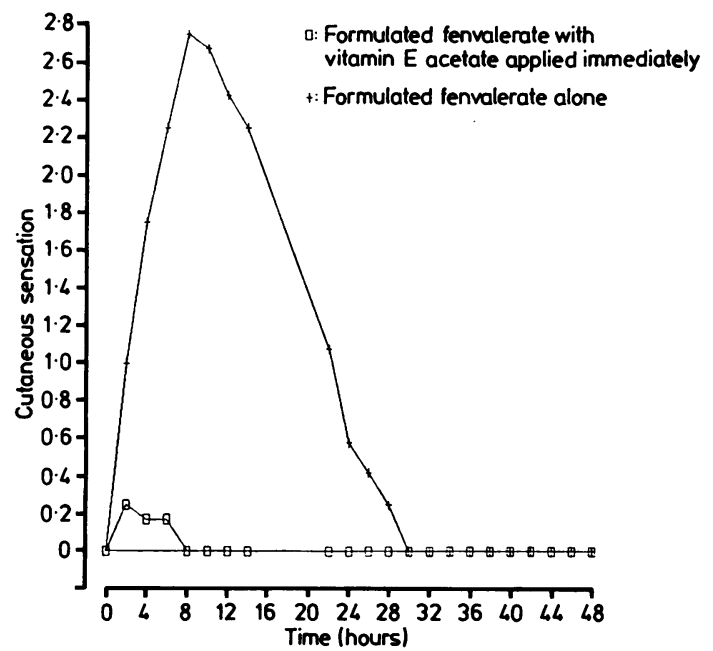

Fig 4 Therapeutic inhibition of formulated fenvalerate $\left(0.13 \mathrm{mg} / \mathrm{cm}^{2}\right)$ with vitamin $E$ acetate $(1 \mathrm{mg}=1 \mathrm{IU})$ on people.

Table 1 Comparison of treatment means for paraesthesia resulting from topical exposure to fenvalerate, permethrin, and the inert ingredients on people

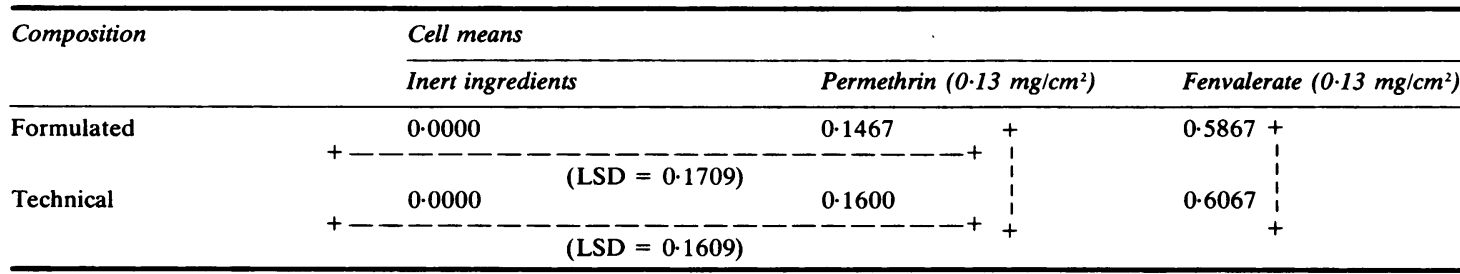

,$+---+:$ Not statistically significant at $p \leqslant 0.05$. 
ure to a synthetic pyrethroid insecticide. Technical and formulated fenvalerate $\left(0.13 \mathrm{mg} / \mathrm{cm}^{2}\right)$ and technical and formulated permethrin $\left(0.13 \mathrm{mg} / \mathrm{cm}^{2}\right)$ were used in this study. An extensive degree of inhibition accompanied the topical application of vitamin $E$ acetate for formulated fenvalerate (fig 4). By comparing the digitised area under each curve, there was an approximate 40 -fold inhibition of cutaneous sensation. Figure 5 presents the therapeutic treatment of technical fenvalerate, with a corresponding 20-fold inhibition of paraesthesia. A statistically significant $(p \leqslant 0.05)$ inhibition of sensation for both purities of fenvalerate is shown by the data in table 2 . The therapeutic index for formulated fenvalerate was substantial at $98 \%$ inhibition, whereas technical fenvalerate reached $100 \%$. Similar results were noted with the cutaneous inhibition of formulated and technical permethrin (figs 6 and 7). In both cases total inhibition of the cutaneous sensation resulted from the topical application of dl-alpha tocopheryl acetate; the vitamin E acetate yielded a therapeutic index of $100 \%$ and a significant difference from either form of permethrin applied separately.

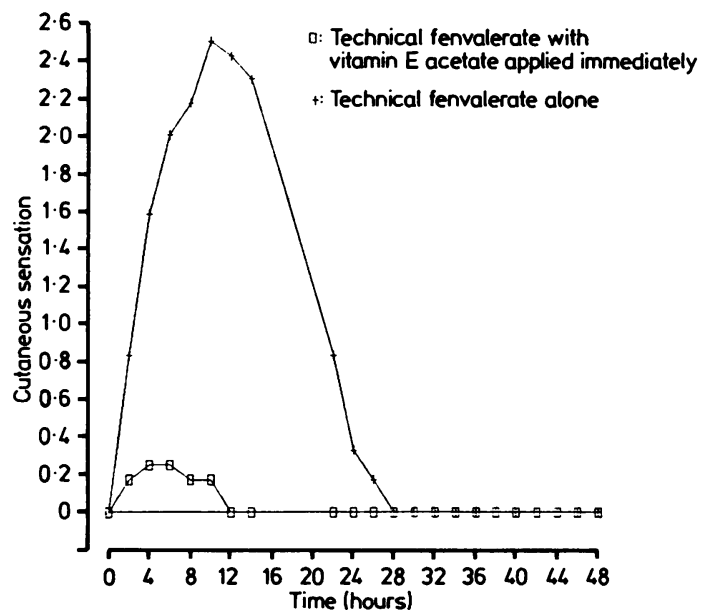

Fig 5 Therapeutic inhibition of technical fenvalerate $\left(0.13 \mathrm{mg} / \mathrm{cm}^{2}\right)$ with vitamin $E$ acetate $(1 \mathrm{mg}=1 \mathrm{IU})$ on people.

\section{Discussion}

All synthetic pyrethroids are lipophilic compounds that are recognised as nerve toxins. They are viscous liquids with relatively high boiling points and low vapour pressures. Since their initial introduction in 1973 , they have attained over $30 \%$ of the commercial market for insecticides by providing an excellent cost/benefit ratio for agricultural and domestic pest control. ${ }^{22}$ Well over 1000 pyrethroids have been synthesised to date.

The biological activity of the synthetic pyrethroids appears to be directly associated with their chemical

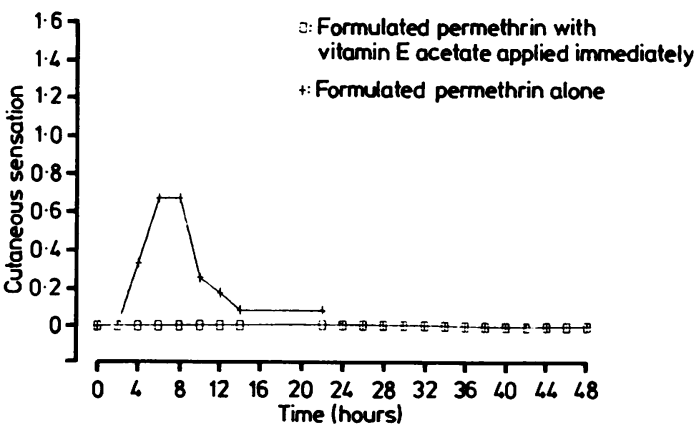

Fig 6 Therapeutic inhibition of formulated permethrin $\left(0.13 \mathrm{mg} / \mathrm{cm}^{2}\right)$ with vitamin $E$ acetate $(1 \mathrm{mg}=1 \mathrm{IU})$ on people.

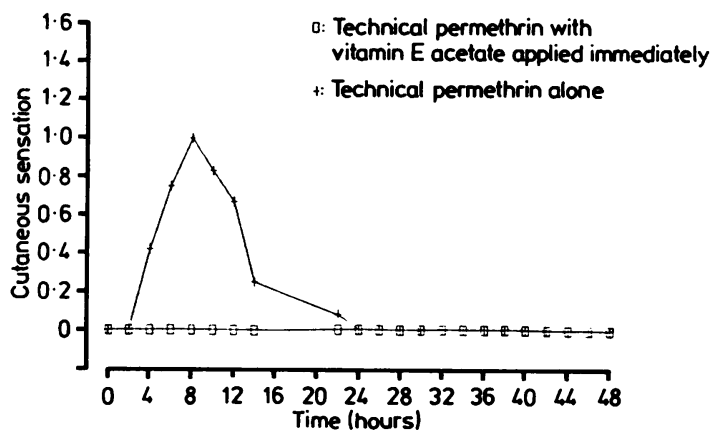

Fig 7 Therapeutic inhibition of technical permethrin $\left(0.13 \mathrm{mg} / \mathrm{cm}^{2}\right)$ with vitamin $E$ acetate $(1 \mathrm{mg}=1 \mathrm{IU})$ on people.

Table 2 Comparison of treatment means for inhibition of paraesthesia by vitamin E acetate $(1 \mathrm{mg}=1 \mathrm{IU})$ on people

\begin{tabular}{lllll}
\hline Synthetic pyrethroid & $\begin{array}{l}\text { Cell mean without vitamin } E \\
\text { acetate }\end{array}$ & $\begin{array}{l}\text { Cell mean with vitamin } E \\
\text { acetate }\end{array}$ & $p$ value & $\begin{array}{c}\text { Therapeutic } \\
\text { index (\%) }\end{array}$ \\
\hline $\begin{array}{l}\text { Formulated fenvalerate } \\
\left(0.13 \mathrm{mg} / \mathrm{cm}^{2}\right)\end{array}$ & 0.6436 & 0.0233 & 0.000 & 98 \\
$\begin{array}{c}\text { Technical fenvalerate } \\
\left(0.13 \mathrm{mg} / \mathrm{cm}^{2}\right)\end{array}$ & 0.5763 & 0.0000 & 0.000 & 100 \\
$\begin{array}{c}\text { Formulated permethrin } \\
\left(0.13 \mathrm{mg} / \mathrm{cm}^{2}\right)\end{array}$ & 0.1236 & 0.0000 & 0.039 & 100 \\
$\begin{array}{c}\text { Technical permethrin } \\
\left(0.13 \mathrm{mg} / \mathrm{cm}^{2}\right)\end{array}$ & 0.1600 & 0.0000 & 0.047 & 100 \\
\hline
\end{tabular}


structure. Almost all active pyrethroids are esters, whose constituent acids and alcohols are basically inert. Electron density and polarisability are of little importance to their reactivity. ${ }^{23}$ The cyano substitute, located in the S-configuration of the 3-phenoxybenzyl alcohol of alpha-cyano synthetic pyrethroids, tends to increase the potency of the molecule by approximately three to sixfold. Fenvalerate has been listed in a range of 0.6 to four times the insecticidal potency of permethrin, depending on the pest species evaluated. ${ }^{12}$ In studies 1 and 2 of this investigation a close correlation was noted between the insecticidal potency (knockdown) for both fenvalerate and permethrin and the degree of induced cutaneous paraesthesia on people.

The human assay, incorporated into studies 1, 2, and 3, necessitated the topical application of milligram quantities of a field strength synthetic pyrethroid to the lobule of a patient's ear. Numerous prior investigations have attested to the safety and efficaciousness of this assay, and the documented low inherent mammalian toxicity of the synthetic pyrethroids. Rapid penetration was enhanced since the pyrethroids are extremely lipophilic and were directly applied to an extremity composed of both areolar and adipose tissue. The auriculotemporal nerve, branching from the inferior maxillary of the trigeminal (fifth cranial) innervates most of the lobule. LeQuesne et al observed in electrophysiological studies that the disturbances produced by the synthetic pyrethroid insecticides occurred more frequently in sensory than in motor nerves and that the symptoms resulting in man from occupational exposures were solely due to sensory nerve involvement. ${ }^{14}$ Based on this rationale, it seemed appropriate for the human assay to use a source of tissue where rapid penetration would be enhanced with prominent sensory nerve involvement.

The cutaneous sensation, often referred to as paraesthesia or dysaesthesia, is the most prominent health symptom that accompanies topical contact with these potent insecticides. ${ }^{13-15}$ It appears to be unique in quality, time of onset, and duration. Previous stinging sensations of the skin have been noted after topical application of certain agents, but generally occurred either immediately or within several minutes after contact and only in a very sensitive subset of the population, usually fair skinned women. ${ }^{24}{ }^{25}$ This is not the case with the synthetic pyrethroid cutaneous sensation, which seems to be almost universal and possess a latent period of one to two hours after application. ${ }^{18}$

Participants in study 1 of this investigation noted a prominent increase in paraesthesia between formulated fenvalerate and formulated permethrin, and between technical fenvalerate and technical per- methrin. Nevertheless, no cutaneous sensation was recorded by any of the same participants on topical exposure to the inert ingredients of these pyrethroids. It would seem that the inert ingredients, organic solvents and surfactants, are not the offending agents causing the paraesthesia. Narahashi, using a voltage clamp assay in combination with various toxins, drugs, and enzymes showed that the nerve impulse, thought to be responsible for this particular sensation, arises from a rapid transient change in the semipermeability of the nerve membrane to sodium and potassium ions. This transition in permeability is governed by molecular pores in the axon membrane referred to as sodium and potassium channels. An extended sodium current, after termination of the nerve membrane depolarisation, is the mechanism thought to be responsible for the occurrence of this repetitive activity. Vijverberg et al have shown that after topical treatment with alpha-cyano pyrethroids, this sodium tail current deteriorates much more gradually than after non-cyano pyrethroids, and that the difference in time restraints is at least one-fold greater. ${ }^{26}$ They also noticed considerably smaller variations in the magnitude of the sodium tail current among agents that belong to the same synthetic pyrethroid category-non-cyano or alpha-cyano. This corresponds closely with the results of study 1 , which did not substantiate a statistically significant difference $(p \geqslant 0.05)$ between the fenvalerate pyrethroids or between the permethrin pyrethroids evaluated at similar concentrations. These results also coincide with an earlier investigation which established that there was no difference between heavy ends and technical fenvalerate, at the same concentration, when evaluated by 36 participants. ${ }^{18}$

In a more recent investigation Vijverberg and van den Bercken concluded that the non-cyano pyrethroids produce sodium tail currents with a time constant of decay in the order of approximately 10 milliseconds, ${ }^{22}$ whereas the alpha-cyano pyrethroids range from hundreds of milliseconds to more than one second. These results were indicative of the fact that the actual rate of relaxation of the activation gate ( $\mathrm{m}$-gate) in pyrethroid affected sodium channels is a characteristic of each particular category of synthetic pyrethroid, and that the rate of relaxation is substantially slower after treatment with alphacyano pyrethroids. Therefore, the rate of relaxation of the sodium channel influx helps to explain the 40-fold difference noted between formulated fenvalerate and formulated permethrin, and between technical fenvalerate and technical permethrin in this investigation.

Study 2 denoted a linear correlation between concentration of a formulated synthetic pyrethroid and 
the degree of induced paraesthesia. Fenvalerate resulted in a substantially steeper slope $(\mathrm{m}=31 \cdot 0)$ than permethrin $(m=15 \cdot 8)$. This could be considered connotative of an enhanced cutaneous sensation from only minor increases in concentration of a formulated alpha-cyano pyrethroid, whereas a non-cyano pyrethroid merely fluctuated to a minor degree over a similar range of concentrations. Possibly, these regression curves are indicative of the fact that, as the concentration of a pyrethroid is increased, so will be the number of sodium channels affected during membrane depolarisation. This would result in a direct influence on the corresponding amplitude of the pyrethroid induced sodium tail current and coincide with the fact that the amplitude of a pyrethroid induced sodium tail current is always proportional to the number of sodium channels that open during nerve membrane depolarisation. ${ }^{22} 2627$

In study 3 vitamin $E$ acetate $\left(\mathrm{C}_{31} \mathrm{H}_{52} \mathrm{O}_{3}\right)$ was found to be a highly efficacious therapeutic agent for synthetic pyrethroid exposure. Vitamin $\mathrm{E}$ acetate (1 $\mathrm{mg}=1 \mathrm{IU}$ ) was chosen because it is a well known biological antioxidant that inhibits peroxides from accumulating and protects cells from the scavenging effects of free radicals. It also ensures the stability and integrity of biological membranes. ${ }^{28}{ }^{29}$ One of the two possible routes of absorption through the epidermis consists of a direct transition through the stratum corneum, granulosum, spinosum, and basalis to the dermis; this is possible since dl-alpha tocopheryl acetate is a highly lipophilic compound. Access is also secured through the pilosebaceous unit and connective tissue sheaths. Dl-alpha tocopheryl acetate also has a high affinity for small blood vessels and possesses an anti-inflammatory capability. Kamimura and Matsuzawa indicate that it will suppress inflammation with either topical or systemic administration, and with prophylactic or therapeutic treatment. ${ }^{20}$ Its anti-inflammatory action is thought to be related to its ability to stabilise lyosomal membranes, histamine liberation from granules of mast cells, and serotonin liberation from tissue cells. Further, vitamin E acetate is nonirritating and non-sensitising when evaluated by itself.

Vitamin $E$ acetate is stable to the oxidising influences of air or light, including ultraviolet light. It is also relatively stable to acids and heat. It may therefore be used practically in the field for occupational exposure with little concern as to handling precautions. To date, no cases of hypervitaminosis $\mathrm{E}$ have been documented from topical application of dl-alpha tocopheryl acetate.

This research was made possible through a grant from the Shell Oil Company.

\section{References}

' Elliott M, Janes NF. Synthetic pyrethroids-a new class of insecticide. Chemical Society Reviews 1978;7:473-505.

${ }^{2}$ Oetting RD, Morishita FS. Potential of pyrethroid insecticides for greenhouse whitefly, Trialeurodes vaporariorum, control. Journal of the Georgia Entomological Society 1980;15:27280.

${ }^{3}$ Elliott M. Synthetic pyrethroids. In: Elliott M, ed. Synthetic pyrethroids. Washington DC: American Chemical Society, 1977:1-28. (American Chemical Society symposium series No 42.)

${ }^{4}$ Elliott M, Farnham W, Janes F, Needham PH, Pulman DA. Insecticidally active conformations of pyrethroids. In: Kohn GK, ed. Mechanism of pesticide action. Washington: American Chemical Society, 1974:80-91. (American Chemical Society symposium series No 2.)

${ }^{5}$ Narahashi T. Nerve membrane as a target for pyrethroids. Pesticide Science 1976;7:267-72.

- Vijverberg HP, van den Bercken J. Frequency-dependent effects of the pyrethroid insecticide decamethrin in frog myelinated nerve fibers. Eur J Pharmacol 1979;58:501-4.

${ }^{7}$ Harris GG, Miline DB. Input-output characteristics of the lateral-line sense organ of Xenopus laevis. J Acoust Soc Am 1966;40:32-41.

${ }^{8}$ van den Bercken J, Akkermans LMS, van der Zalm JM. DDTlike action of allethrin in the sensory nervous system of Xenopus laevis. Eur J Pharmacol 1973;21: 195-206.

${ }^{4}$ Miyamoto J. Degradation, metabolism and toxicity of synthetic pyrethroids. Environ Health Perspec 1976;14:15-28.

${ }^{10}$ Hutson DH. The metabolic fate of pyrethroid insecticides in mammals. Progress in Drug Metabolism 1979;3:215-52.

" Elliott M. The future for insecticides. In: Locke M, Smith DS, eds. Insect biology in the future. New York: Academic Press, 1980:879-903.

12 Casida JE. Pyrethrum flowers and pyrethroid insecticides. Environ Health Perspec 1980;34:189-202.

${ }^{13}$ Kolmodin-Hedman B, Swensson A, Akerblom M. Occupational exposure to some synthetic pyrethroids (permethrin and fenvalerate). Arch Toxicol 1982;50:27-33.

14 LeQuesne PM, Maxwell UC, Butterworth ST. Transient facial sensory symptoms following exposure to synthetic pyrethroids: a clinical and electrophysiological assessment. Neurotoxicol 1980;2:1-11.

is Tucker SB, Flannigan SA. Cutaneous effects from occupational exposure to fenvalerate. Arch Toxicol 1983;54:195-202.

${ }^{16}$ Martin TJ, Hester KH. Dermatitis caused by insecticidal pyrethrum flowers (Chrysanthemum cinerariaelfolium). Br J Dermatol Syph 1941;53:127-42.

${ }^{17}$ Knox JM, Tucker SB. A new cutaneous sensation caused by synthetic pyrethroids. Clin Res 1982;30:915A.

${ }^{18}$ Knox JM, Tucker SB, Flannigan SA. Paresthesia from cutaneous exposure to a synthetic pyrethroid insecticide. Arch Dermatol 1984; 120:744-6.

${ }^{14}$ Winer BJ. Single-factor experiments having repeated measures on the same elements. In: Winer BJ, ed. Statistical principles in experimental design. New York: McGraw-Hill, 1962:105-40.

${ }^{20}$ Engelman L. One-way analysis of variance and covariance. In: Dixon WJ, ed. BMDP statistical software. Berkeley: University of California Press, 1981:345-436.

21 Snedecor GW, Cochran WG. One-way classifications; analysis of variance. In: Snedecor GW, Cochran WG, eds. Statistical methods. Ames, Iowa: Iowa State University Press, 1980:215-39.

${ }^{22}$ Vijverberg HP, van den Bercken J. Action of pyrethroid insecticides on the vertebrate nervous system. Neuropathol Appl Neurobiol 1982;8:421-40.

${ }^{23}$ Elliott M, Janes NF, Potter C. The future of pyrethroids in insect control. Ann Rev Entomol 1978;23:443-69.

${ }^{24}$ Laden K. Studies on irritancy and stinging potential. Journal of 
the Society of Cosmetic Chemists 1973;24:385-93.

${ }^{25}$ Frosch PH, Kligman AM. A method for appraising the stinging capacity of topically applied substances. Journal of the Society of Cosmetic Chemists 1977;28:197-209.

${ }^{26}$ Vijverberg HP, van der Zalm JM, van Kleef RG, van den Bercken J. Temperature and structure dependent interaction of pyrethroids with the sodium channels in frog node of Ranvier. Biochimica et Biophysica Acta 1983;728:73-82.

${ }^{27}$ Beeman RW. Recent advances in mode of action of insecticides.
Ann Rev Entomol 1982;27:253-81.

${ }^{28}$ de Duve C, Wattiaux R, Wibo M. Effects of fat-soluble compounds on lysosomes in vitro. Biochem Pharmacol 1962;9:97-116.

${ }^{24}$ Ehrlich HP, Taver H, Hunt TK. Inhibitory effects of vitamin E on collagen synthesis and wound repair. Ann Surg 1972;175:235-40.

${ }^{30}$ Kamimura M, Matsuzawa T. Percutaneous absorption of alpha tocopheryl acetate. J Vitaminol 1968;14:150-9.

\section{The May 1985 issue}

\section{THE MAY 1985 ISSUE CONTAINS THE FOLLOWING PAPERS}

\section{Editorial}

"One simple set of regulations"

Neurobehavioural effects of repeated occupational exposure to toluene and paint solvents NICOLA CHERRY, HELEN HUTCHINS, T PACE, AND HA WALDRON

No acute behavioural effects of exposure to styrene: a safe level of exposure? C EDLING AND KERSTIN EKBERG

Dechlorination of halocarbons by microsomes and vesicular reconstituted cytochrome $\mathrm{P}-450$ systems under reductive conditions A G SALMON, J A NASH, C M WALKLIN, AND R B FREEDMAN

Clinical and immunological reactions to Aspergillus niger among workers at a biotechnology plant $M D$ TOPPING, D A SCARISBRICK, CM LUCZYNSKA, E C CLARKE, AND A SEATON

A study of dose-response relationships for asbestos associated disease M M FINKELSTEIN

Rates of sickness absenteeism among employees of a modern hospital: the role of demographic and occupational factors A PINES, K SKULKEO, E POLLAK, E PERITZ, AND J STEIF
Accident liability J B KUNÉ

Risk factors for radiogenic cancer: a comparison of factors derived from the Hanford survey with those recommended by the ICRP K F BAVERSTOCK AND D G PAPWORTH

Some factors influencing interobserver variation in classifying simple pneumoconiosis D C MUSCH, I T T HIGGINS, AND J R LANDIS

\section{Short reports}

Asymmetric rapidly progressive lung fibrosis: a cause of pseudotumour in asbestosis WOCM COOKSON, J J GLANCY, AND F A FROST

Reticulocyte haem synthesis in occupational exposure to trinitrotoluene H SAVOLAINEN, R TENHUNEN, AND HA HÄRKÖNEN

Book reviews

Correction: Health of workers exposed to electric fields (February 1985)

Information section

Copies are still available and may be obtained from the PUBLISHING MANAGER, BRITISH MEDICAL ASSOCIATION, TAVISTOCK SQUARE, LONDON WC1H 9JR, price $£ 4 \cdot 25$ (USA \$9.20), including postage. 